\title{
The moment problem on divisible abelian semigroups
}

\author{
Nobuhisa SAKAKIBARA \\ (Received July 20, 1988)
}

\section{Introduction}

The moment problem is concerned with the integral representation of positive definite functions on semigroups. A recent detailed study of the moment problem is found in [2]. The purpose of this paper is to prove that every positive definite function on a divisible countable semigroup admits a unique integral representation.

Let $S$ be an abelian semigroup with zero element 0 . A semicharacter on $S$ is a function $\rho: S \rightarrow \boldsymbol{R}$ such that $\rho(0)=1, \rho(s+t)=\rho(s) \rho(t)$ for all $s$, $t \in S$. The set $S^{*}$ of all semicharacters on $S$ is called the dual semigroup of $S$. We equip $S^{*}$ with the topology of pointwise convergence. A function $\varphi: S \rightarrow \boldsymbol{R}$ is called positive definite if

$$
\sum_{i, j=1}^{n} c_{i} c_{j} \varphi\left(s_{i}+s_{j}\right) \geqq 0
$$

for all $n \in \boldsymbol{N},\left\{s_{1}, \cdots, s_{n}\right\} \subset S$ and $\left\{c_{1}, \cdots, c_{n}\right\} \subset \boldsymbol{R}$. A function $\psi: S \rightarrow \boldsymbol{R}$ is called negative definite if

$$
\sum_{i, j=1}^{n} c_{i} c_{j} \psi\left(s_{i}+s_{j}\right) \leqq 0
$$

for all $n \in \boldsymbol{N},\left\{s_{1}, \cdots, s_{n}\right\} \subset S$ and $\left\{c_{1}, \cdots, c_{n}\right\} \subset \boldsymbol{R}$ with $\sum_{i=1}^{n} c_{i}=0$. Let $M_{+}\left(S^{*}\right)$ denote the set of all nonnegative Radon measures on $S^{*}$, and let $E_{+}\left(S^{*}\right)$ denote the set of $\mu \in M_{+}\left(S^{*}\right)$ such that

$$
\int_{S^{*}}|\rho(s)| d \mu(\rho)<\infty \text { for all } s \in S \text {. }
$$

A function $f: S \rightarrow \boldsymbol{R}$ is called a moment function if there exists a measure $\mu \in E_{+}\left(S^{*}\right)$ such that

$$
f(s)=\int_{S^{*}} \rho(s) d \mu(\rho) \text { for } s \in S .
$$

Every moment function is positive definite. It is known (see [4]) that every bounded positive definite function is a moment function whose re- 
presenting measure is unique. But a positive definite function is not necessarily a moment function, and also a representing measure for a moment function is not necessarily unique. For instance, according to the classical Hamburger moment problem, every positive definite function on the additive semigroup of nonnegative integers $N_{0}$ is a moment function, but there exists a positive definite function whose representing measure is not unique.

An abelian semigroup $S$ is called perfect if every positive definite function is a moment function whose representing measure is unique. For instance, the additive semigroup of nonnegative rational numbers $\boldsymbol{Q}_{+}$is perfect (see [2], Proposition 6.5.6). Prefect semigroups form a rather restrictive class, while they have some very nice properties:

(1) The direct sum of a countable family of perfect semigroups is perfect (see [2], Note VI).

(2) Any homomorphic image of a perfect semigroup is perfect (see [2], Theorem 6.5.5).

An abelian semigroup $S$ is called 2-divisible if every $s \in S$ can be written $s=t+t$ for some $t \in S$.

Berg [1] proved the following results.

THEOREM A. The abelian semigroup $(\boldsymbol{D},+)$ of dyadic numbers (i.e. $\left.\boldsymbol{D}=\left\{k 2^{-n} \mid k, n \in \boldsymbol{N}_{0}\right\}\right)$ is perfect.

THEOREM B. If a countable abelian semigroup $S$ is 2-divisible, then $S$ is perfect.

We say that an abelian semigroup $S$ is divisible if every $s \in S$ can be written $s=n t$ for some $n \geqq 2$ and some $t \in S$. In $\S 2$ of this paper, we shall generalize the above Berg's results to the wider class of divisible abelian semigroups. In $\S 3$, we shall characterize the completely negative difinite functions on a divisible abelian semigroup by the notion of Schur monotonicity.

\section{Main results}

For each sequence $\vec{m}=\left\{m_{n}\right\}_{n \geqq 1}$ of integers $m_{n} \geqq 2$, we define the abelian semigroup

$$
T(\vec{m})=\left\{\frac{k}{m_{1} \cdots m_{n}} \mid k \in N_{0}, n \geqq 1\right\} .
$$

As particular cases, we have $T(\vec{m})=\boldsymbol{Q}_{+}$if $m_{n}=n+1$ for $n \geqq 1$, and $T(\vec{m})$ $=\boldsymbol{D}$ if $m_{n}=2$ for $n \geqq 1$. We shall prove that $(T(\vec{m}),+)$ is perfect for each $\vec{m}$. 
First, we consider the case when $m_{n}$ is odd for every $n \geqq 1$. For $x \in$ $\boldsymbol{R}$ the function $\rho_{x}: T(\vec{m}) \longrightarrow \boldsymbol{R}$ defined by $\rho_{x}(r)=e^{r x}$ is a semicharacter and so is $\rho_{-\infty}:=\mathbf{1}_{\{0\}}$, the indicator function of $\{0\}$. Since each $m_{n}$ is odd, the function $x\left(\frac{k}{m_{1} \cdots m_{n}}\right):=(-1)^{k}$ is well defined and multiplicative on $T(\vec{m})$. Then $x \rho_{x}$ is also a semicharacter for $x \in \boldsymbol{R}$. Note that $\chi=\boldsymbol{1}_{2 T(\vec{m})}$ $-\boldsymbol{1}_{T(\vec{m}) \backslash 2 T(\vec{m})}$. Conversely let $\rho \in T(\vec{m})^{*}$. Then $\rho(1) \in \boldsymbol{R}$ and $x=\log |\rho(1)| \in$ $\underline{\boldsymbol{R}}(:=[-\infty, \infty))$. It is easy to see that, for $r=\frac{k}{m_{1} \cdots m_{n}} \in T(\vec{m}), \rho(r)=$ $\rho(1)^{r}$ if $\rho(1) \geqq 0$ and $\rho(r)=(-1)^{k}(-\rho(1))^{r}$ if $\rho(1)<0$. Hence $\rho=\rho_{x}$ if $\rho(1) \geqq 0$ and $\rho=x \rho_{x}$ if $\rho(1)<0$. Moreover the mapping $\rho \longmapsto \rho(1)$ is a topological semigroup isomorphism of $T(\vec{m})^{*}$ onto $(\underline{\boldsymbol{R}}, \cdot)$. Thus we may identify $T(\vec{m})^{*}$ with $\underline{\boldsymbol{R}}$ and also with the disjoint union $\underline{\boldsymbol{R}} \cup \boldsymbol{R}$.

THEOREM 2.1. Let $\vec{m}=\left\{m_{n}\right\}_{n \geqq 1}$ be a sequence of odd numbers greater than 2. Then the semigroup $(T(\vec{m}),+)$ is perfect. Every positive definite function $\varphi$ on $T(\vec{m})$ has a unique representation

$$
\varphi(r)=a \mathbf{1}_{\{0\}}(r)+\int_{R} e^{r x} d \mu(x)+\int_{R} x(r) e^{r x} d \nu(x)
$$

for all $r \in T(\vec{m})$, where $a \geqq 0$ and $\mu, \nu \in M_{+}(\boldsymbol{R})$ satisfy

$$
\int_{R} e^{r x} d \mu(x)<\infty, \int_{R} e^{r x} d \nu(x)<\infty \text { for } r \in T(\vec{m}) .
$$

PROOF : Let $\ell_{n}=m_{1} \cdots m_{n}$ for $n \geqq 1$. Let $\varphi$ be a positive definite function on $T(\vec{m})$. For each $n \geqq 1,\left\{\varphi\left(\frac{k}{\ell n}\right)\right\}_{k \geqq 0}$ is a Hamburger moment sequence because $k \longmapsto \varphi\left(\frac{k}{\ell_{n}}\right)$ is positive definite on $\left(\boldsymbol{N}_{0},+\right)$. Therefore it follows (see [2], Theorem 6.2.2) that there exists a $\mu_{n} \in M_{+}(\boldsymbol{R})$ such that

$$
\begin{aligned}
& \int_{R}|x|^{k} d \mu_{n}(x)<\infty \text { for } k \geqq 0, \\
& \varphi\left(\frac{k}{\ell_{n}}\right)=\int_{R} x^{k} d \mu_{n}(x) \text { for } k \geqq 0 .
\end{aligned}
$$

Define the mappings $f_{n}: \underline{\boldsymbol{R}} \longrightarrow[0, \infty)$ and $g_{n}: \underline{\boldsymbol{R}} \longrightarrow(-\infty, 0]$ by

$$
f_{n}(x)=\exp \left(\frac{x}{\ell_{n}}\right), g_{n}(x)=-\exp \left(\frac{x}{l_{n}}\right) \text { for } x \in \underline{\boldsymbol{R}} \text {. }
$$

Then $f_{n}$ and $g_{n}$ are homeomorphisms, so there exist $\tau_{n}, \sigma_{n} \in M_{+}(\underline{\boldsymbol{R}})$ such that $\tau_{n}^{\circ} f_{n}^{-1}=\mu_{n} \mid[0, \infty)$ and $\sigma_{n}^{\circ} g_{n}^{-1}=\mu_{n} \mid(-\infty, 0)$. Hence we have 


$$
\varphi\left(\frac{k}{\ell_{n}}\right)=\int_{\underline{R}} \exp \left(\frac{k}{\ell_{n}} x\right) d \tau_{n}(x)+\int_{\underline{R}} x\left(\frac{k}{\ell_{n}}\right) \exp \left(\frac{k}{\ell_{n}} x\right) d \sigma_{n}(x) .
$$

Since $\tau_{n}(\underline{\boldsymbol{R}})+\sigma_{n}(\underline{\boldsymbol{R}})=\varphi(0)<\infty,\left\{\tau_{n}\right\}_{n \geq 1}$ and $\left\{\sigma_{n}\right\}_{n \geq 1}$ are relatively compact in the vague topology on $M_{+}(\underline{\boldsymbol{R}})$ (see [2], Proposition 2.4.6). Since the vague topology on $M_{+}(\underline{\boldsymbol{R}})$ is metrizable (see [2], Proposition 2.4.10), there is an increasing sequence $n_{1}<n_{2}<\cdots$ such that $\tau_{n_{i}}$ and $\sigma_{n_{i}}$ converge vaguely to $\tau, \sigma \in M_{+}(\underline{\boldsymbol{R}})$, respectively, with total masses uniformly bounded by $\varphi(0)$.

Let $r=\frac{k}{l_{n}} \in T(\vec{m})$ be fixed. For $i \geqq 1$ such that $n_{i} \geqq n$, we have

$$
\begin{aligned}
\varphi(r) & =\varphi\left(k m_{n+1} \cdots m_{n_{i}} / \ell_{n_{i}}\right) \\
& =\int_{\underline{R}} e^{r x} d \tau_{n_{i}}(x)+\int_{\underline{\underline{R}}} x(r) e^{r x} d \sigma_{n_{i}}(x) .
\end{aligned}
$$

Using the fact that, for each nonnegative continuous function $f$, the integral $\int f d \mu$ is lower semicontinuous in $\mu$ with respect to the vague topology (see [2], p. 50), we have

$$
\begin{aligned}
& \int_{\underline{R}} e^{2 r x} d \tau(x) \leqq \liminf _{i \rightarrow \infty} \int_{\underline{R}} e^{2 r x} d \tau_{n_{i}}(x) \leqq \varphi(2 r), \\
& \int_{\underline{R}} e^{2 r x} d \sigma(x) \leqq \liminf _{i \rightarrow \infty} \int_{\underline{R}} e^{2 r x} d \sigma_{n_{i}}(x) \leqq \varphi(2 r) .
\end{aligned}
$$

Since $e^{r x} \leqq\left(1+e^{2 r x}\right) / 2, e^{r x}$ is integrable with respect to $\tau$ and $\sigma$. Let $h(x)$ $=1+e^{2(r+1) x}$ for $x \in \underline{\boldsymbol{R}}$. Since the sequence $\left\{h(x) \tau_{n_{i}}\right\}_{i \geqq 1}$ converges vaguely to $h(x) \tau$, with total masses bounded uniformly by $\varphi(0)+\varphi(2(r+1))$ and since $e^{r x} / h(x) \in C_{0}(\underline{\boldsymbol{R}})$, it follows (see [2], Proposition 2.4.4) that

$$
\begin{aligned}
\lim _{i \rightarrow \infty} \int_{\underline{R}} e^{r x} d \tau_{n_{i}}(x) & =\lim _{i \rightarrow \infty} \int_{\underline{R}} \frac{e^{r x}}{h(x)} h(x) d \tau_{n_{i}}(x) \\
& =\int_{\underline{R}} \frac{e^{r x}}{h(x)} h(x) d \tau(x) \\
& =\int_{\underline{R}} e^{r x} d \tau(x) .
\end{aligned}
$$

Similarly

$$
\lim _{i \rightarrow \infty} \int_{\underline{\underline{R}}} e^{r x} d \sigma_{n_{i}}(x)=\int_{\underline{\underline{R}}} e^{r x} d \sigma(x) .
$$

Since $r \in T(\vec{m})$ is arbitrary, we have

$$
\varphi(r)=\int_{\underline{R}} e^{r x} d \tau(x)+\int_{\underline{R}} x(r) e^{r x} d \sigma(x) \text { for all } r \in T(\vec{m}) .
$$


Defining $a=\tau(\{-\infty\})+\sigma(\{-\infty\}), \mu=\tau \mid(-\infty, \infty)$ and $\nu=\sigma \mid(-\infty, \infty)$ we have

$$
\varphi(r)=a \mathbf{1}_{\{0\}}(r)+\int_{R} e^{r x} d \mu(x)+\int_{R} x(r) e^{r x} d \nu(x),
$$

which shows that $\varphi$ is a moment function on $(T(\vec{m}),+)$.

Next we prove the uniqueness of the triple $(a, \mu, \nu)$. Since

$$
\lim _{r \rightarrow 0} \varphi(2 r)=\mu(\boldsymbol{R})+\nu(\boldsymbol{R})=\varphi(0)-a,
$$

$a$ is uniquely determined by $\varphi$. Suppose that $\mu^{\prime}, \nu^{\prime} \in M_{+}(\boldsymbol{R})$ satisfy

$$
\varphi(r)=a \mathbf{1}_{\{0\}}(r)+\int_{R} e^{r x} d \mu^{\prime}(x)+\int_{R} x(r) e^{r x} d \nu^{\prime}(x)
$$

for all $r \in T(\vec{m})$. Then, for $r \in 2 T(\vec{m})$, we have

$$
\int_{R} e^{r x} d(\mu+\nu)(x)=\int_{R} e^{r x} d\left(\mu^{\prime}+\nu^{\prime}\right)(x) .
$$

Define the function $\Phi$ on the closed right half-plane $C_{+}=\{z \in C \mid \operatorname{Re} z \geqq 0\}$ by

$$
\Phi(z)=\int_{R} e^{z x} d\left(\mu+\nu-\mu^{\prime}-\nu^{\prime}\right)(x),
$$

which is well defined and continuous on $\boldsymbol{C}_{+}$and holomorphic in its interior. Since $2 T(\vec{m})$ is dense in $[0, \infty), \Phi(z)=0$ for $\operatorname{Re} z>0$ by uniqueness theorem, so that $\Phi\left(e^{i y}\right)=0$ for $y \in \boldsymbol{R}$ by continuity. By the injectivity of Fourier-Stieltjes transform (see [5], p. 17), we have $\mu+\nu-\mu^{\prime}-\nu^{\prime}=0$. Since

$$
\int_{R} e^{r x} d(\mu-\nu)(x)=\int_{R} e^{r x} d\left(\mu^{\prime}-\nu^{\prime}\right)(x)
$$

for $r \in T(\vec{m}) \backslash 2 T(\vec{m})$, by the similar argument we have $\mu-\nu-\mu^{\prime}+\nu^{\prime}=0$. Therefore $\mu=\mu^{\prime}$ and $\nu=\nu^{\prime}$. Thus the triple $(a, \mu, \nu)$ is unique.

Secondly, we consider the case when $\left\{m_{n} \mid n \in N\right\} \cap 2 \boldsymbol{N}$ is nonvoid and finite. Assume that $m_{p}$ is even and $m_{n}$ is odd for all $n>p$, and let $\iota=$ $m_{1} \cdots m_{p}$. In this case, the function $\chi=\boldsymbol{1}_{2 T(\vec{m})}-\boldsymbol{1}_{T(\vec{m}) \backslash 2 T(\vec{m})}$ is given by $\chi$ $\left(\frac{k}{m_{1} \cdots m_{n}}\right)=(-1)^{k}$ where $n \geqq p$, so that $x$ is multiplicative. Then the functions $\rho_{x}(r)=e^{r x}(x \in \boldsymbol{R}), \rho_{-\infty}=\boldsymbol{1}_{\{0\}}$ and $x \rho_{x}(x \in \boldsymbol{R})$ are semicharacters. Conversely let $\rho \in T(\vec{m})^{*}$. Then it is easy to see that, for $r=\frac{k}{m_{1} \cdots m_{n}}$ where $n \geqq p, \rho(r)=\rho(1)^{r}$ if $\rho\left(\frac{1}{\iota}\right) \geqq 0$ and $\rho(r)=(-1)^{k}(-\rho(1))^{r}$ if $\rho\left(\frac{1}{\iota}\right)$ 
$<0$. Hence $\rho=\rho_{x}$ if $\rho\left(\frac{1}{\ell}\right) \geqq 0$ and $\rho=x \rho_{x}$ if $\rho\left(\frac{1}{\ell}\right)<0$, where $x=\log$ $\rho(1) \in \underline{\boldsymbol{R}}$. The mapping $\rho \longmapsto \rho\left(\frac{1}{\ell}\right)$ is a topological semigroup isomorphism of $T(\vec{m})^{*}$ onto $(\boldsymbol{R}, \cdot)$. Thus we may identify $T(\vec{m})^{*}$ with $\boldsymbol{R}$ and also with the disjoint union $\underline{\boldsymbol{R}} \cup \boldsymbol{R}$. Just as before, we can prove that Theorem 2.1 remains valid for this case.

Finally, we consider the case when $\left\{m_{n} \mid n \in N\right\} \cap 2 \boldsymbol{N}$ is infinite. Note in this case that $T(\vec{m})$ is 2-divisible, so that it is perfect by Theorem $B$. Moreover, the set of semicharacters are $\left\{\rho_{x}\right\}_{x \in \underline{R}}$ and we identify $T(\vec{m})^{*}$ with $\underline{\boldsymbol{R}}$. Hence, for every positive definite function $\varphi$ on $T(\vec{m})$, we have a unique representation

$$
\varphi(r)=a \mathbf{1}_{\{0\}}(r)+\int_{\boldsymbol{R}} e^{r x} d \mu(x)
$$

for all $r \in T(\vec{m})$, where $a \geqq 0$, and $\mu \in M_{+}(\boldsymbol{R})$ satisfies

$$
\int_{R} e^{r x} d \mu(x)<\infty \text { for } r \in T(\vec{m}) .
$$

Consequently, we have the next theorem.

THEOREM 2.2. Let $\vec{m}=\left\{m_{n}\right\}_{n \geqq 1}$ be a sequence of integers $m_{n} \geqq 2$. Then the semigroup $(T(\vec{m}),+)$ is perfect.

Using this theorem and the properties (1) and (2) stated in $\S 1$, we have the following.

THEOREM 2.3. Every countable divisible abelian semigroup $S$ is perfect.

Proof: Suppose $S=\left\{0, s_{1}, s_{2}, \cdots\right\}$. Since $S$ is divisible, for every $s_{j}$ there exist a sequence $\vec{m}^{(j)}=\left\{m_{n}^{(j)}\right\}_{n \geq 1}$ of integers $m_{n}^{(j)} \geqq 2$ and a sequence $\left\{t_{n}^{(j)}\right\}_{n \geq 1}$ of elements in $S$ such that

$$
s_{j}=m_{1}^{(j)} t_{1}^{(j)}, t_{n}^{(j)}=m_{n+1}^{(j)} t_{n+1}^{(j)} \text { for } n \geqq 1 .
$$

Note that for $r=k / m_{1}^{(j)} \cdots m_{n}^{(j)} \in T\left(\vec{m}^{(j)}\right)$ the element $r s_{j}:=k t_{n}^{(j)}$ is well defined. We define the mapping $\pi: \bigoplus_{j=1}^{\infty} T\left(\vec{m}^{(j)}\right) \longrightarrow S$ by

$$
\pi\left(r_{1}, r_{2}, \cdots\right)=\sum_{j=1}^{\infty} r_{j} s_{j}
$$

Then $\pi$ is a surjective homomorphism. Every $T\left(\vec{m}^{(j)}\right)$ is perfect by Theorem 2.2, so that $\bigoplus_{j=1}^{\infty} T\left(\vec{m}^{(j)}\right)$ is perfect by (1) in $\S 1$. Hence $S=$ 
$\pi\left(\bigoplus_{j=1}^{\infty} T\left(\vec{m}^{(j)}\right)\right)$ is prefect by (2) in $\S 1$. This completes the proof.

We further give the next theorem concerning the integral representation of negative definite functions on $(T(\vec{m}),+)$. The proof can be done by modifying that in [2, proposition 6.5.13], for the integral representation of negative definite functions on $\left(\boldsymbol{Q}_{+},+\right)$.

THEOREM 2.4. Let $\vec{m}=\left\{m_{n}\right\}_{n \geqq 1}$ be a sequence of integers $m_{n} \geqq 2$. Let $\psi$ be a negative definite function on $T(\vec{m})$.

(i) If $\left\{m_{n} \mid n \in \boldsymbol{N}\right\} \cap 2 \boldsymbol{N}$ is finite, then $\psi$ has a form

$$
\begin{aligned}
\psi(r)=a & +b r-c r^{2}+d \mathbf{1}_{\{0\}}(r) \\
& +\int_{R \backslash\{0\}}\left(1-e^{r x}-r\left(1-e^{x}\right)\right) d \mu(x) \\
& +\int_{R}\left(1-\chi(r) e^{r x}\right) d \nu(x),
\end{aligned}
$$

where $a, b \in \boldsymbol{R}, c, d \geqq 0, \mu \in M_{+}(\boldsymbol{R} \backslash\{0\})$ and $\nu \in M_{+}(\boldsymbol{R})$ satisfy

$$
\begin{aligned}
& \int_{0<|x| \leq 1} x^{2} d \mu(x)<\infty, \\
& \int_{|x|>1} e^{r x} d \mu(x)<\infty, \int_{R} e^{r x} d \nu(x)<\infty \text { for } r \in T(\vec{m}) .
\end{aligned}
$$

The sextuple $(a, b, c, d, \mu, \nu)$ is uniquely determined by $\psi$.

(ii) If $\left\{m_{n} \mid n \in \boldsymbol{N}\right\} \cap 2 \boldsymbol{N}$ is infinite, then $\psi$ has a form

$$
\begin{aligned}
\psi(r)=a & +b r-c r^{2}+d \mathbf{1}_{\{0\}}(r) \\
& +\int_{\boldsymbol{R} \backslash\{0\}}\left(1-e^{r x}-r\left(1-e^{x}\right)\right) d \mu(x),
\end{aligned}
$$

where $a, b \in \boldsymbol{R}, c, d \geqq 0, \mu \in M_{+}(\boldsymbol{R} \backslash\{0\})$ satisfies

$$
\begin{aligned}
& \int_{0<|x| \leq 1} x^{2} d \mu(x)<\infty, \\
& \int_{|x|>1} e^{r x} d \mu(x)<\infty \quad \text { for } \quad r \in T(\vec{m}) .
\end{aligned}
$$

The quintuple $(a, b, c, d, \mu)$ is uniquely determined by $\psi$.

\section{Application to Schur monotonicity}

In this section, applying Theorem 2.2, we characterize the completely negative definite functions on a divisible abelian semigroup in terms of Schur monotonicity.

Let $A$ be a convex subset of some real linear space $E$. For two vectors $x=\left(x_{1}, \cdots, x_{n}\right)$ and $y=\left(y_{1}, \cdots, y_{n}\right)$ in $E^{n}$ whose components $x_{i}$ and 
$y_{i}$ belong to $A$, we say $x$ is majorized by $y$ and write $x<y$ if there exists an $n \times n$ doubly stochastic matrix $P=\left(p_{i j}\right)$ such that

$$
x_{i}=\sum_{j=1}^{n} p_{i j} y_{j} \quad \text { for } i=1, \cdots, n .
$$

Let $S$ be an abelian semigroup. A function $\psi: S \longrightarrow \boldsymbol{R}$ is called completely negative definite if $\psi(\cdot+a)$ is negative definite for all $a \in S$. For each $n \in \boldsymbol{N}$, a function $\psi: S \longrightarrow \boldsymbol{R}$ is called Schur increasing of order $n$ if, for every $\nu=\left(\nu_{1}, \cdots, \nu_{n}\right)$ and $\mu=\left(\mu_{1}, \cdots, \mu_{n}\right)$ in $\operatorname{Mol}_{+}^{1}(S)^{n}$ such that $\nu<\mu$, the inequality

$$
\int \psi d\left(\nu_{1} * \cdots * \nu_{n}\right) \leqq \int \psi d\left(\mu_{1} * \cdots * \mu_{n}\right)
$$

holds, where $\operatorname{Mol}_{+}^{1}(S)$ denotes the set of all Radon probability measures with finite support.

Note (see [2, Chapter 7]) that a function $\psi: S \longrightarrow \boldsymbol{R}$ is Schur increasing of order 2 if and only if $\psi$ is negative definite, and that if $\psi$ is Schur increasing of order $n \geqq 3$, then $\psi$ is completely negative difinite. Conversely, Berg [1] proved the following.

THEOREM C. Let $S$ be a 2-divisible abelian semigroup. Then every negative definite function on $S$ is Schur increasing of all orders.

The next theorem extends Theorem $C$ to the case of a divisible abelian semigroup. Here we note that a negative definite function on a divisible abelian semigroup is not necessarily completely negative definite (for example, $\psi\left(k 3^{-n}\right)=-(-1)^{k}$ on $\left\{k 3^{-n} \mid k \in N_{0}, n \geqq 1\right\}$ ).

THEOREM 3.1. Let $S$ be a divisible abelian semigroup. Then every completely negative definite function on $S$ is Schur increasing of all orders.

PROOF: Let $\psi$ be a completely negative definite function on $S$. Let $\mu=\left(\mu_{1}, \cdots, \mu_{n}\right)$ and $\nu=\left(\nu_{1}, \cdots, \nu_{n}\right)$ in $\operatorname{Mol}_{+}^{1}(S)^{n}$ be given such that $\nu<\mu$. There exists a finite set $F \subset S$ on which all $\mu_{i}$ (and hence all $\nu_{i}$ ) are concentrated. Suppose $F=\left\{s_{1}, \cdots, s_{d}\right\}$. Since $S$ is divisible, for every $s_{j}$ there exists a sequence $\vec{m}^{(j)}=\left\{m_{n}^{(j)}\right\}_{n \geq 1}$ of integers $m_{n}^{(j)} \geqq 2$ and a sequence $\left\{t_{n}^{(j)}\right\}_{n \geq 1}$ in $S$ such that

$$
s_{j}=m_{1}^{(j)} t_{1}^{(j)}, t_{n}^{(j)}=m_{n+1}^{(j)} t_{n+1}^{(j)} \text { for } n \geqq 1 .
$$

Let $S_{0}$ be a subsemigroup of $S$ generated by $\left\{t_{n}^{(j)} \mid n \geqq 1, j=1,2, \cdots, d\right\}$. Then $S_{0} \supset F$. It is seen as in the proof of Theorem 2.3 that $S_{0}$ becomes a homomorphic image of $\bigoplus_{j=1}^{\infty} T\left(\vec{m}_{i}^{(j)}\right)$. Hence $S_{0}$ is perfect by Theorem 2.2 
and (1), (2) in $\S 1$. Since every completely negative definite function on a perfect abelian semigroup is Schur increasing of all orders (see [2], Theorem 7.3.9), $\psi^{\prime}=\psi \mid S_{0}$ is Schur increasing of all orders as a function on $S_{0}$, and hence

$$
\int \psi^{\prime} d\left(\nu_{1} * \cdots * \nu_{n}\right) \leqq \int \psi^{\prime} d\left(\mu_{1} * \cdots * \mu_{n}\right) .
$$

Since $\mu_{1} * \cdots * \mu_{n}$ and $\nu_{1} * \cdots * \nu_{n}$ are concentrated on $S_{0}$, we have

$$
\int \psi d\left(\nu_{1} * \cdots * \nu_{n}\right) \leqq \int \psi d\left(\mu_{1} * \cdots * \mu_{n}\right),
$$

which shows that $\psi$ is Schur increasing of order $n$.

REMARK. After completing the paper, the author has known that Bisgaard and Ressel [3] recently proved a more general result than Theorem 2.3 by a different method.

The author would like to express his hearty thanks to Professors T. Ando, F. Hiai and Y. Nakamura for their helpful suggestions and encouragement.

\section{References}

[1] C. BERG, Fonctions définies négatives et majoration de Schur. Lecture Notes in Math. No. 1096, Springer-Verlag, Berlin-Heidelberg-New York, 1984, pp. 69-89.

[2] C. Berg, J. P. R. CHRISTENSEN and P. RESSEL, Harmonic Analysis on Semigroups, Springer-Verlag, New York-Berlin-Heidelberg-Tokyo, 1984.

[ 3 ] T. M. BISGAARD and P. RESSEL, Unique disintegration of arbitrary positive definite functions on *-divisible semigroups, Math. Z. 200 (1989), 511-525.

[4] R. J. LINDAHL, and P.H. MASERICK,'Positive-definite functions on involution semigroups, Duke Math. J. 38 (1971), 771-782.

[ 5 ] W. RUDIN, Fourier Analysis on Groups, Interscience Publishers, New York, 1967.

Division of Applied Mathematics

Research Institute of Applied Electricity

Hokkaido University

Sapporo 060, Japan 